\title{
A FATAL PULMONARY INFECTION BY NOCARDIA BRASILIENSIS
}

\author{
*V Wadhwa, S Rai, P Kharbanda, S Kabra, R Gur, VK Sharma
}

\begin{abstract}
The reported case is of primary pulmonary nocardiosis, caused by Nocardia brasiliensis, in a immunocompromised patient, which ended fatally despite appropriate treatment. The partially acid fast filamentous bacterium was predominant on direct examination of the sputum. It was cultured on blood agar, MacConkey agar and by paraffin baiting technique. The bacterium was resistant to cotrimoxazole, the drug of choice for nocardiosis.
\end{abstract}

Key words: Nocardiosis, cotrimoxazole resistant, Nocardia brasiliensis

Pulmonary nocardiosis, a chronic suppurative infection occurring in immunosuppressed patients, can be self limited, transient or subclinical and it may progress to an acute, subacute or chronic illness mimicking tuberculosis, mycotic infection or a malignancy.

$N$. brasiliensis is the second most common clinically isolated actinomycete and is usually associated with localized cutaneous infections. ${ }^{1}$ Cotrimoxazole, the antimicrobial agent of choice against nocardiosis is highly effective against this species.The reported case was unusual, in being a fatal primary pulmonary infection caused by $N$. brasiliensis resistant to cotrimoxazole.

\section{Case Report}

A 42 year - old male vegetable vendor presented with history of breathlessness, fever and cough since five days. The patient was earlier diagnosed as a case of chronic obstructive airway disease (COAD) and was receiving oral steroids since one year.

On examination his temperature was $37.2^{\circ} \mathrm{C}$; pulse, 108/min.; BP, 110/80 $\mathrm{mmHg}$ and respiratory rate, $40 / \mathrm{min}$. Cyanosis, clubbing and pedal edema were present. Auscultation revealed bilateral lower zone and midzonal crepts and wheeze. No abnormality was detected on examination of abdomen and CNS. Chest radiograph showed bilateral infiltrates and patchy consolidation. Blood and sputum samples were sent for laboratory investigations and empirical treatment with intravenous ceftriaxone ( $2 \mathrm{~g} \mathrm{OD})$, amikacin (400 mg OD) and ceftazidime (2 g TDS) was started.

Laboratory studies revealed: haemoglobin, $11.8 \mathrm{~g} / \mathrm{dL}$; total leucocyte count, $17000 / \mathrm{mm}^{3}$ and erthrocyte sedimentation rate

*Corresponding author (e-mail: < vishal2870@yahoo.com>) Department of Microbiology, Maulana Azad Medical College and LN hospital, New Delhi - 110 002, India

Received: 07-01-05

Accepted: 02-03-05 of $85 \mathrm{~mm}$ in the first hour. Blood culture was sterile after seven days of aerobic incubation. The sputum had an acceptable Bartlett count and showed predominant gram positive, thin, beaded, filamentous bacteria with right angle branching resembling Nocardia spp. ${ }^{2}$ The bacterium was acid fast with $1 \% \mathrm{H}_{2} \mathrm{SO}_{4}$ (Fig. 1).

Repeat samples yielded similar findings. Sample was

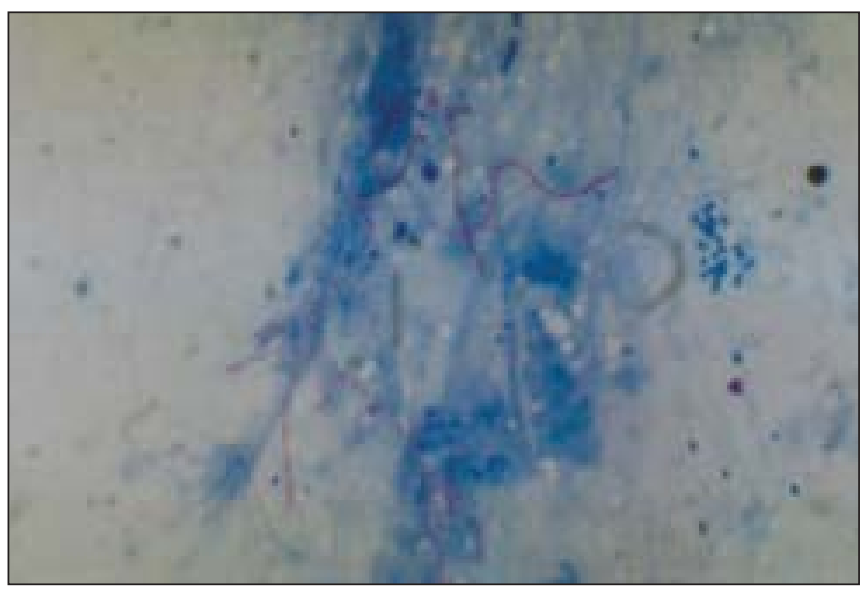

Figure 1: Photomicrograph of sputum showing acid fast, beaded filamentous bacteria with typical right angle branching (x 1000)

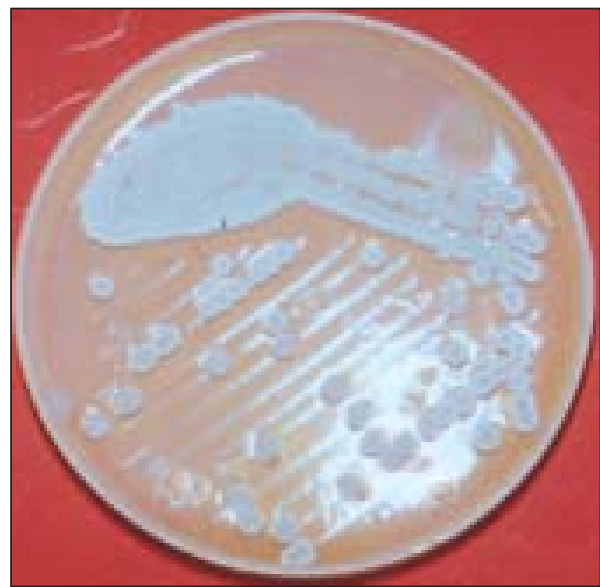

Figure 2: White colonies of $N$. brasiliensis on nutrient agar 
cultured by using paraffin baiting technique and on to blood and MacConkey agar. ${ }^{3}$ After overnight incubation, growth of commensal bacteria was observed. Subsequent examination after 72 hours revealed white pigmented dry colonies with rough surface on the solid media and in paraffin bait at the aerobic junction. Examination of these cultures on staining revealed resemblance to Nocardia species. The growth was sub cultured on nutrient agar for better appreciation of pigment production (Fig. 2).

The bacterium was confirmed as Nocardia spp. on the basis of morphology and standard biochemical tests. ${ }^{2}$ On disk diffusion test the isolate was sensitive to amikacin, gentamicin, chloramphenicol and resistant to erythromycin, ceftriaxone, ampicillin, cotrimoxazole and ciprofloxacin. ${ }^{4}$

The bacterium was identified as $N$. brasiliensis / N. pseudobrasiliensis by its ability to hydrolyze casein and utilize myo-inositol as the sole source of carbon. ${ }^{5}$ Resistance to ciprofloxacin confirmed the organism to be $N$. brasiliensis. ${ }^{6}$

\section{Discussion}

Pulmonary nocardiosis is a rare infection with high fatality rate $(62 \%)$. Prognostically this infection can be divided into two groups, one receiving immunosuppressive antineoplastic or corticosteroid therapy (mortality $80-100 \%$ ) and the other group constitutes previously healthy patients and those with untreated underlying disease (mortality 15-20\%). The difference results from the extensive pulmonary necrosis (not dissemination) in those receiving immunosuppressants. ${ }^{7}$

Pulmonary infection due to $N$. brasiliensis is almost always associated with direct extension of the mycetoma through the chest wall. ${ }^{8}$ Cotrimoxazole, the drug of choice for nocardiosis has excellent response against $N$. brasiliensis, $N$. asteroides and $N$. transvalensis whereas, $N$. farcinica and $N$. nova show $7 \%$ and $11 \%$ resistance respectively. It is not recommended for treatment of $N$. otitidiscaviarum infections due to high resistance among isolates. ${ }^{9}$

Our patient was immmunocompromised and suffered a primary pulmonary infection due to $N$. brasiliensis resistant to cotrimoxazole. The case highlights the usefulness of the antimicrobial sensitivity testing in clinical isolates of Nocardia and infers that, till we diagnose such cases early on in the disease progression by routine examination, the result of this disease will remain grave.

\section{References}

1. Mc Neil MM, Brown JM. The medically important aerobic Actinomycetes, epidemiology and microbiology. Clin Microbiol Rev 1994;7:357-17.

2. Brown JM, McNeil MM, Desmond EP. Nocardia, Rhodococcus, Gordona, Actinomadura, Streptomyces and other actinomycetes of medical importance. In: Murray PR, Baron EJ, Pfaller MA, Tenover FC and Yolken RH, eds. Manual of Clinical Microbiology. (American Society of Microbiology: Washington, DC); 1999. p. 370-98.

3. Ayyar S, Tendolkar U, Deodhar L. A Comparison of three media for isolation of Nocardia species from clinical specimens. $J$ Postgrad Med 1992;38:70-2.

4. Wallace RJ, Septimus EJ, Musher DM, Martin RR. Disk diffusion susceptibility testing of Nocardia species. J Infect Dis 1977;135:568-76.

5. The Aerobic Gram-Positive Bacilli. In: Koneman EW, Allen SD, Janda WM, Schreckenberger and Winn WC, editors. Color Atlas and Textbook of Diagnostic Microbiology. Lippincott-Raven: Philadelphia; 1997. p. 651-708.

6. Wallace RJ, Brown BA, Blacklock Z, Ulrich R, Jost K, Brown $\mathrm{JM}$, et al. New Nocardia taxon among isolates of Nocardia brasilienisis associated with invasive disease. J Clin Microbiol 1995;33:1528-33.

7. Presant CA, Serpick AA. Factors affecting survival in Nocardiosis. Am Rev Respir Dis 1971;103:882-3.

8. Causey WA, Sieger B. Systemic nocardiosis caused by Nocardia brasiliensis. Am Rev Respir Dis 1974;109:134-7.

9. Lerner PI. Nocardiosis. Clinical Infect Dis 1996;22:891-905. 